\title{
Prognostic impact of lymph node harvest for patients with node-negative esophageal squamous cell carcinoma: a large-scale multicenter study
}

\author{
Qi-Xin Shang ${ }^{1}$, Yu-Shang Yang ${ }^{1}$, Li-Yan $\mathrm{Xu}^{2}$, Hong Yang ${ }^{3}$, Yin $\mathrm{Li}^{4}$, Yi $\mathrm{Li}^{4}$, Zhi-Yong $\mathrm{Wu}^{2}$, Jian-Hua Fu ${ }^{3}$, \\ Xiao-Dong Yao ${ }^{2}$, Xiu-E Xü ${ }^{2}$, Jian-Yi Wu ${ }^{2}$, Long-Qi Chen ${ }^{1}$ \\ ${ }^{1}$ Department of Thoracic Surgery, West China Hospital of Sichuan University, Chengdu, China; ${ }^{2}$ Department of Oncology Surgery, Shantou \\ Affiliated Hospital, Sun Yat-sen University, Guangzhou, China; ${ }^{3}$ Department of Thoracic Oncology, Sun Yat-sen University Cancer Center, \\ Guangzhou, China; ${ }^{4}$ Department of Thoracic Surgery, Cancer Hospital Chinese Academy of Medical Sciences, Beijing, China \\ Contributions: (I) Conception and design: LQ Chen, Y Li, ZY Wu, JH Fu, XE Xu, JY Wu, YS Yang, QX Shang; (II) Administrative support: LY Xu, \\ H Yang; (III) Provision of study materials or patients: LY Xu, Y Li, ZY Wu, JH Fu, JY Wu, LQ Chen; (IV) Collection and assembly of data: QX \\ Shang, YS Yang, XE Xu, JY Wu; (V) Data analysis and interpretation: QX Shang, LQ Chen; (VI) Manuscript writing: All authors; (VII) Final \\ approval of manuscript: All authors. \\ Correspondence to: Prof. Long-Qi Chen, MD, PhD. Department of Thoracic Surgery, West China Hospital of Sichuan University, No. 37, Guoxue \\ Alley, Chengdu 610041, China. Email: drchenlq@scu.edu.cn.
}

Background: We examined the association between the number of resected lymph nodes and survival to determine the optimal lymphadenectomy for thoracic esophageal squamous cell carcinoma (ESCC) patients with negative lymph node.

Methods: We included 1,836 patients from Chinese three high-volumed hospitals with corresponding clinicopathological characters such as gender, age, tumor location, tumor grade and TNM stage of patients. The median follow-up of included patients was 45.7 months (range, 1.03-117.3 months). X-Tile plot was used to identify the lowest number of lymphadenectomy. The multivariate model's construction was in use of parameters with clinical significance for survival and a nomogram based on clinical variable with $\mathrm{P}<0.05$ in Cox regression analysis. Both two models were validated using a cohort extracted from the Surveillance, Epidemiology, and End Results (SEER) 18 registries database between 1975 and 2016 (n=951).

Results: More lymphadenectomy numbers were significantly associated with better survival in patients both in training cohort [hazard ratio $(\mathrm{HR})=0.980 ; 95 \%$ confidence interval $(\mathrm{CI}): 0.971-0.988 ; \mathrm{P}<0.001$ ] and validation cohort $(\mathrm{HR}=0.980 ; 95 \% \mathrm{CI}: 0.968-0.991 ; \mathrm{P}=0.001)$. Cut-off point analysis determined the lowest number of 9 for thoracic ESCC patients in N0 stage through training cohort (C-index: 0.623; sensitivity: $80.7 \% ; 1$ - specificity: $72.5 \%$ ) when compared with 10 in validation cohort (C-index: 0.643; sensitivity: $78.2 \% ; 1$ - specificity: $63.0 \%$ ). The cut-off points of 9 were examined in training cohort and validated in the divided cohort from validation cohort (all $\mathrm{P}<0.05$ ). Meanwhile, nomograms for both cohorts were constructed and the calibration curves for both cohorts agreed well with the actual observations in terms of predicting 3- and 5-year survival, respectively.

Conclusions: Larger number for lymphadenectomy was associated with better survival in thoracic ESCC patients in N0 stage. Nine was what we got as the lowest number for lymphadenectomy in pN0 ESCC patients through this study, and our result should be confirmed further.

Keywords: Esophageal squamous cell carcinoma (ESCC); node-negative patients; optimal lymphadenectomy number; prognosis

Submitted Sep 04, 2020. Accepted for publication Jul 28, 2021.

doi: 10.21037/jgo-20-371

View this article at: https://dx.doi.org/10.21037/jgo-20-371 


\section{Introduction}

Esophageal cancer (EC) is considered as one of the most malignant cancer with nearly 40,000 deaths every year all over the world, accounting for $4.9 \%$ of all cancer caused deaths $(1,2)$. Similarly, number of deaths cases correlated with EC ranked at 4th place among all the tumor-related deaths in China (3). Previous studies have demonstrated the prognostic value of tumor infiltration (T stage), lymph node metastasis ( $\mathrm{N}$ stage) and distant metastasis ( $\mathrm{M}$ stage), being assembled in American Joint Committee on Cancer (AJCC) staging manual to define the final stage of EC. Besides, cancer location, histologic grade and histopathologic cell type, providing potential association with prognosis and tumor development were also added in manual from the 7 th edition $(4,5)$. Among these prognostic factors, lymph node metastasis (LNM) was regarded as the most important prognostic factor correlated with the prognosis in esophageal squamous cell carcinoma (ESCC) patients (4-6). Lymphadenectomy has been widely accepted to restrict and eliminate the LNM. Basically, the recommendation of resecting 10 regional lymph nodes for pT1 cancers, 20 for $\mathrm{pT} 2$, and $\leq 30$ for $\mathrm{pT} 3$ in terms of AJCC cancer staging manual 8 th ed is regarded as the current clinical guideline for lymphadenectomy (6); however, what should be the lowest number to use for lymph node resection in EC remains on discussion (7-10). Some scholar suggested that extensive lymphadenectomy increased morbidity and might also increase perioperative mortality rather than significantly improve patients' survival $(11,12)$. However, others held the opinion that wider lymph node dissection guaranteed the elimination of known or occult disease as well as micrometastases, and thereby lead to improved prognosis in EC patients $(9,13,14)$. Meanwhile, some studies reported that patients without lymph node metastasis also developed locoregional recurrence, which may be attributed to the presence of nodal micrometastases (NMMs) (15-18).

There is a controversy regarding the lowest number recommended for lymphadenectomy during esophagectomy in pNO ESCC patients and very few studies addressed this issue. Therefore, this study aimed to evaluate the prognostic significance of the dissected number of lymph nodes in pN0 ESCC patients. To this end, we evaluated patient data from three high-volume Chinese hospitals including various regions and clinical practice, validating the outcomes through SEER database, which may represent real world situation more accurately and help further determine the lowest threshold required for the number of resected lymph nodes. We present the article in accordance with the TRIPOD reporting checklist (available at https://dx.doi. org/10.21037/jgo-20-371).

\section{Methods}

\section{Patients}

We retrospectively reviewed two patient cohorts. One cohort (training cohort) comprised 1836 ESCC patients from three high-volumed hospitals: the West China Hospital, Shantou University Medical College, and Sun Yat-sen University Cancer Centre who underwent radical esophagectomy from April 2008 to April 2012. patients were ruled out in line with the criteria as follows: (I) patients who were lost to follow-up; (II) other types of EC confirmed pathologically; (III) patients without receiving R0 resection; (IV) patients with more than one positive lymph nodes; (V) the number of resected lymph node is zero; (VI) patients with receiving pre-or postoperative adjuvant therapy; (VII) patients with cervical EC or esophagogastric junction carcinoma; (VIII) patients with other malignant tumors; (IX) patients who were defined as M1 preoperatively. The study was conducted in accordance with the Declaration of Helsinki (as revised in 2013), and was approved by the human participants' committee of West China Hospital of Sichuan University (No. 2021762A), Shantou University Medical College (No. 2020-094), and Sun Yat-sen University Cancer Centre (No. 2013B021800163, Science and Technology Plan Projects of Guangdong Province of China), and the written consents of operation and the application of the resected specimens were obtained preoperatively. To validate results obtained in our study and compare them with those of a different cohort, we obtained patients data for the second cohort (validation cohort) from the Surveillance, Epidemiology, and End Results (SEER) cancer registry.

\section{SEER database}

A total of 32,872 patients with ESCC diagnosed from 1975 to 2016 were extracted from the latest version of the SEER database released in April 2019 (covering 18 registries) using SEER*Stat software (seer.cancer.gov/seerstat) Version 8.3.6 (19). We retrieved the individual data of each patient from SEER database, including diagnostic age, sex, survival months, histology types, vital status recode, grade, primary tumor site, AJCC $\mathrm{T} 7$ th ed, AJCC N 7 th ed, 


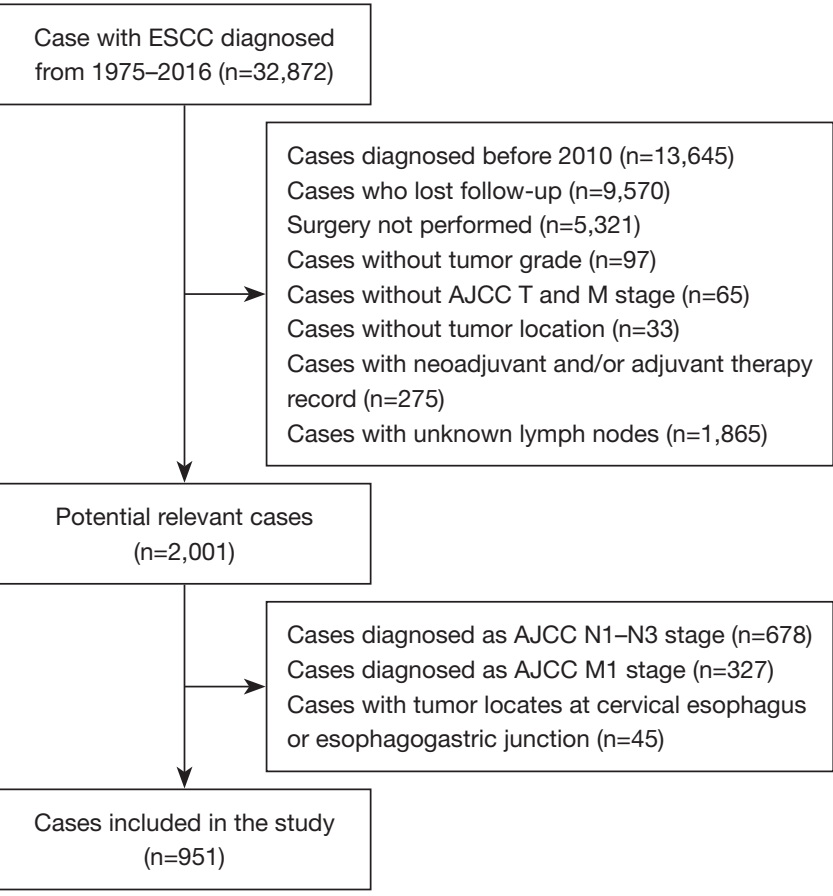

Figure 1 The details and the process of selection of patients data extracted from the SEER database. ESCC, esophageal squamous cell carcinoma; AJCC, American Joint Committee on Cancer; SEER, Surveillance, Epidemiology, and End Results.

AJCC $M$ 7th ed, radiation record, chemotherapy record, regional nodes examined as well as regional nodes positive. Included EC patients who were defined by the International Classification of Diseases for Oncology (ICD-O-3)/World Health Organization 2009 site code C153-C155 were regarded as eligible cases. Estimated overall survival (OS) recorded in the SEER database was the "cause-specific classification of death", and was stratified as "dead (attributable to this cancer dx)" or "alive or dead of other cause". Meanwhile, we excluded patients who met the exclusion criteria of our study. What needs to be emphasized is that the patients who were diagnosed before 2010 were also excluded from this study owing to the lack of specific AJCC 7th staging system until 2010. The details and the process of selection of patients' data extracted from SEER database are shown in Figure 1. Finally, 951 patients were included in validation cohort of our study.

\section{Surgery and pathology}

In terms of patients' preoperative examinations and general condition, the Mckeown, the Sweet or the Ivor-Lewis esophagogastrectomy with radical lymphadenectomy was selected individually. Meanwhile, anastomoses followed by esophagogastrectomy was performed safely and normally. Additionally, the gastric conduit was the preferred means of reconstruction for the most of surgeons.

The surgeon separated lymph nodes from the resected esophagus and para-esophageal tissues. The specimens were routinely fixed, embedded and stained by means of formalin, diaminobenzidine chromogen counterstained solution and hematoxylin subsequently. The standard method of histological examination of each specimen was conducted by two experienced pathologists, at the same time, the pathologists also recorded the extent and the station of metastatic lymph nodes during the examination.

\section{Follow up}

In this study, patients were followed up every 3 months within 2 years postoperatively and the pan will be changed as every 6 months from the third to the fifth year. After the fifth year, the patients will be followed up every 1 year. The postoperative examinations such as chest CT, gastroscopy, blood test and neck or abdominal ultrasound will be recommended for patients. Information about the patients including tumor stage, recurrence, metastasis as well as survival status will be recorded through outpatient, telephone or letter follow up.

\section{Statistical analysis}

The clinicopathological characteristics of patients in both cohorts included were analyzed by the means of the Pearson's chi-squared test or Fisher's exact test to compare the dichotomous variables, and the Student's $t$-test was applied for the mean values of continuous variables agreed to normality distribution assumption, others were analyzed through Mann-Whitney U test. We used the X-Tile plot (http://www.tissuearray.org) to identify the lowest number of lymphadenectomy in training cohort and compared it with the lowest number obtained through the analysis of the validation cohort (20). OS in patients in both cohorts was calculated by Kaplan-Meier curves and the log-rank tests based on the lowest number in training cohort was used to determine the statistical significance. Cox proportional hazard regression model was used for multivariate survival analysis. With the help of the X-Tile plot, we also performed stratified analyses by $\mathrm{T}$ stage in both cohorts in 
order to make the comparison. The statistical significance was regarded as the probability value $<0.05$ in two-sided test, and all the statistical analysis were conduct by $\mathrm{IBM}^{\circledR}$ SPSS ${ }^{\circledR}$ Statistics Version 21.0.

\section{Nomogram construction}

The nomogram with variables obtained from the analysis of training cohort was built using R Programming Language $\left(\mathrm{R}^{\circledR}\right.$ Version 3.4.0, http://www.r-project.org/) and we validated it with validation cohort. The nomogram model for prognosis was employed to predict 3- and 5-year OS in thoracic ESCC patients without lymph node metastasis using the variables obtained with variables obtained in multivariate analysis using in Cox proportional hazard regression model, and $\mathrm{P}$ value $<0.05$ was defined as statistically significant. The nomograms of two cohorts were evaluated from two aspects: on the one hand, the discrimination of nomogram will be assessed by means of concordance index (C-index); on the other hand, we compared the observed and the predicted survival rates with the corresponding 3- and 5-year cancer-specific survival probabilities calculated by the nomogram. The average of 200 bootstrap samples' Kaplan-Meier estimates was used to quantify the bootstrap-corrected 3- and 5-year OS.

\section{Results}

\section{Patient characteristics and the distribution of resected lymph nodes}

After applying selection criteria, 1,836 thoracic ESCC patients from Chinese centers and 951 patients whose data was extracted from SEER database, were enrolled in this study. The clinicopathological characteristics of both cohorts are listed in Table 1. Overall, the median number of resected lymph nodes was 14 (range, 1-78; mean 15.57; SD 9.28 ) in training cohort while, among patients in validation cohort, the median number of resected was 14 (range, 1-87; mean 15.21; SD 10.14). The median follow-up for the patients in training cohort, was 45.7 months (range, $1.03-117.3$ months), and 3- and 5-year survival rates were $66.6 \%$ and $58.2 \%$, respectively. For validation cohort, the median follow-up time was 33.3 months (range, 0.883.7 months), and the 3 - and 5 -year survival rates were $67.8 \%$ and $58.7 \%$, respectively. The distribution of the number of lymph nodes resected in both cohorts are presented in Figure 2.

\section{Impact of lymphadenectomy numbers on survival}

When the variables of age, sex, tumor location, differentiation, $\mathrm{T}$ stage were used for Cox regression analysis, we found that higher lymphadenectomy values were significantly associated with better survival both in training cohort [hazard ratio $(\mathrm{HR})=0.980 ; 95 \%$ confidence interval (CI): $0.971-0.988 ; \mathrm{P}<0.001]$ and in validation cohort (HR $=0.980 ; 95 \%$ CI: 0.968-0.991; $\mathrm{P}=0.001)$. After stratification by age, sex, tumor location, differentiation and $\mathrm{T}$ stage, the differences for both cohorts remained significant in subgroups of lower thoracic ESCC, patients older than 55 years old, gender, grade of G2 and G3 as well as $\mathrm{T} 2$ and $\mathrm{T} 3$ stages (Table 2).

\section{Impact of the least lymphadenectomy number on survival}

The cut-off point analyses were performed through X-tile in training cohort and the lowest lymphadenectomy number in thoracic ESCC patients without metastatic lymph nodes was defined as 9. Among patients in training cohort, those with $>9$ lymph nodes resected had significantly better prognosis when compared with patients with $\leq 9$ lymph nodes resected $(\mathrm{P}<0.001$, Figure 3$)$. The variables tested in univariate analysis, showed that the factors significantly associated with OS were age, differentiation, tumor location, lymphadenectomy number, and $\mathrm{T}$ stage. In multivariate analysis, Age ( $\mathrm{HR}=1.334$; 95\% CI: $1.123-$ 1.584; $\mathrm{P}=0.001)$, differentiation $(\mathrm{HR}=1.212 ; 95 \% \mathrm{CI}$ : 1.079-1.361; $\mathrm{P}=0.001$ ), tumor location ( $\mathrm{HR}=0.864 ; 95 \%$ CI: $0.767-0.973 ; \mathrm{P}=0.016$ ), lymphadenectomy number (HR $=0.733 ; 95 \%$ CI: $0.618-0.869 ; \mathrm{P}=0.000)$, and T stage (HR $=1.591 ; 95 \% \mathrm{CI}: 1.445-1.751 ; \mathrm{P}=0.000)$ were found as independent prognostic factors for OS (Table 3).

\section{Validation of prognostic impact of number 9 for resected lymph nodes in validation cohort}

We then used patient data from validation cohort to validate the prognostic impact of the number obtained. Patients in validation cohort were divided into two groups according to the lowest number of lymphadenectomy ( $\mathrm{n}=9$ ). Log-rank tests showed that the OS in the group of patients with $>9$ lymph nodes resected was significantly better than that in patients with $\leq 9$ lymph nodes resected $(\mathrm{P}<0.001$, Figure 4). Variables used in Cox regression analysis included sex, age, Differentiation, tumor location, lymphadenectomy number, and $\mathrm{T}$ stage. Age (HR $=1.560$; $95 \% \mathrm{CI}: 1.113-$ 
Table 1 Clinicopathological characters of training cohort and validation cohort

\begin{tabular}{|c|c|c|c|}
\hline & Training cohort $(n=1,836)$ & Validation cohort $(n=951)$ & $P$ value \\
\hline Male & 1,393 & 748 & \\
\hline Female & 443 & 203 & \\
\hline Age & & & $<0.001$ \\
\hline$\geq 55$ years & 1,279 & 820 & \\
\hline Grade & & & $<0.001$ \\
\hline Well & 316 & 243 & \\
\hline Moderate & 948 & 400 & \\
\hline Upper & 280 & 72 & \\
\hline Middle & 1,115 & 181 & \\
\hline Lower & 441 & 698 & \\
\hline T stage & & & $<0.001$ \\
\hline $\mathrm{T} 1$ & 357 & 437 & \\
\hline $\mathrm{T} 2$ & 393 & 156 & \\
\hline T3 & 958 & 332 & \\
\hline $\mathrm{T} 4$ & 128 & 26 & \\
\hline
\end{tabular}

SEER, Surveillance, Epidemiology, and End Results; LD number, lymphadenectomy number.

A

$$
120
$$

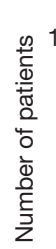

120

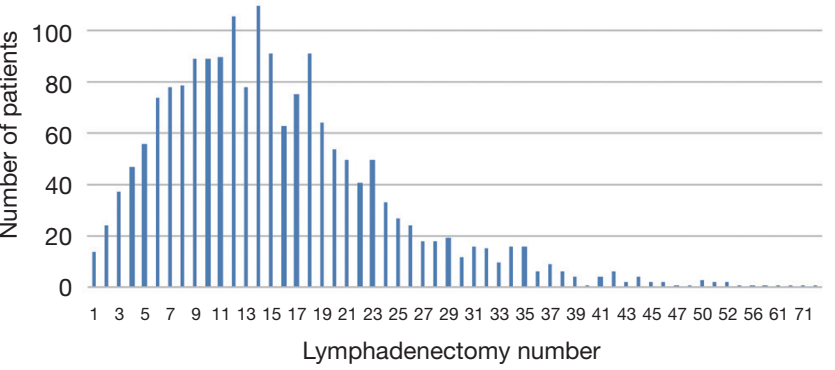

B

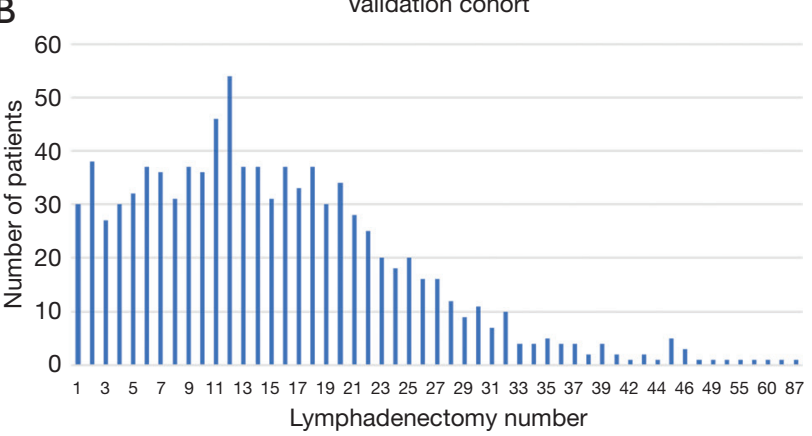

Figure 2 Distribution of the lymphadenectomy number in patients with thoracic esophageal squamous cell carcinoma (ESCC) from the (A) cohort from three tertiary esophagus centers (Training cohort) and the (B) Surveillance, Epidemiology, and End Results cohort (validation cohort). 
Table 2 Cox regression analysis of lymphadenectomy number on overall survival in two cohorts

\begin{tabular}{|c|c|c|c|c|c|c|}
\hline & \multicolumn{3}{|c|}{ Overall survival of training cohort } & \multicolumn{3}{|c|}{ Overall survival of validation cohort } \\
\hline LD summary & 0.980 & $0.971-0.988$ & $<0.001$ & 0.980 & $0.968-0.991$ & 0.001 \\
\hline \multicolumn{7}{|l|}{ Sex } \\
\hline Male & 0.979 & $0.969-0.989$ & $<0.001$ & 0.981 & $0.968-0.994$ & 0.005 \\
\hline \multicolumn{7}{|l|}{ Age } \\
\hline$<55$ years & 0.985 & $0.968-1.002$ & 0.093 & 0.992 & $0.954-1.031$ & 0.690 \\
\hline$\geq 55$ years & 0.977 & $0.967-0.988$ & $<0.001$ & 0.978 & $0.966-0.990$ & $<0.001$ \\
\hline \multicolumn{7}{|l|}{ Differentiation } \\
\hline Low & 0.976 & $0.961-0.992$ & 0.003 & 0.957 & $0.927-0.987$ & 0.005 \\
\hline \multicolumn{7}{|l|}{ Location } \\
\hline Upper & 0.989 & 0.969-1.009 & 0.269 & 0.997 & $0.975-1.020$ & 0.814 \\
\hline Middle & 0.979 & $0.968-0.990$ & $<0.001$ & 0.994 & $0.971-1.018$ & 0.634 \\
\hline Lower & 0.970 & $0.949-0.993$ & 0.009 & 0.967 & $0.952-0.983$ & $<0.001$ \\
\hline \multicolumn{7}{|l|}{ T stage } \\
\hline $\mathrm{T} 1$ & 0.989 & $0.959-1.019$ & 0.472 & 0.985 & $0.966-1.004$ & 0.119 \\
\hline $\mathrm{T} 2$ & 0.966 & $0.945-0.988$ & 0.002 & 0.967 & $0.937-0.998$ & 0.039 \\
\hline
\end{tabular}

SEER, Surveillance, Epidemiology, and End Results; LD, lymphadenectomy; HR, hazard ratio; 95\% Cl, 95\% confidence interval; Ref, Reference.

2.188; $\mathrm{P}=0.010)$, differentiation $(\mathrm{HR}=1.246 ; 95 \% \mathrm{CI}$ : $1.078-1.441 ; \mathrm{P}=0.003)$, tumor location $(\mathrm{HR}=0.728 ; 95 \%$ CI: $0.626-0.847 ; \mathrm{P}<0.001$ ), lymphadenectomy number (HR $=0.611 ; 95 \%$ CI: $0.491-0.760 ; \mathrm{P}<0.001)$ and $\mathrm{T}$ stage $(\mathrm{HR}$ $=1.371 ; 95 \%$ CI: $1.228-1.530 ; \mathrm{P}<0.001)$ were found as the independent prognostic factors for OS (Table 3).

\section{Comparison between lowest numbers and subgroup analyses}

We next performed a cut-off point analysis in validation cohort in order to test whether the lowest number of lymphadenectomies was consistent between the two cohorts, and 10 was found to be the lowest number in validation cohort, which was similar to the number 9 found in training cohort. Meanwhile, the lymphadenectomy number of 10 was also demonstrated as an independent prognostic factor for OS in patients of the validation cohort $(\mathrm{HR}=0.572$; 95\% CI: 0.463-0.706; $\mathrm{P}<0.001)$. Then, we stratified patients by $\mathrm{T}$ stage in order to assess what was the lowest number of lymphadenectomies for every $\mathrm{T}$ stage. Regarding the distribution of lymphadenectomy number at different $T$ stages, the median number in training cohort was 13 (range, $1-52$; mean 14.63; SD 8.50) in contrast to 14 (range, 1-65; mean 15.58; SD 10.12) of validation cohort in patients with $\mathrm{T} 1$ stage $(\mathrm{P}=0.151)$. In patients with $\mathrm{T} 2$ stage, the median resected number in training cohort was 14 (range, 1-56; mean 15.05; SD 8.96), compared with 12 (range, 1-60; mean 14.17; SD 9.94) in validation cohort $(\mathrm{P}=0.319)$. For $\mathrm{T} 3$ stage, the median number of lymphadenectomies in training cohort and validation cohort was 15 (range, 1-78; mean 

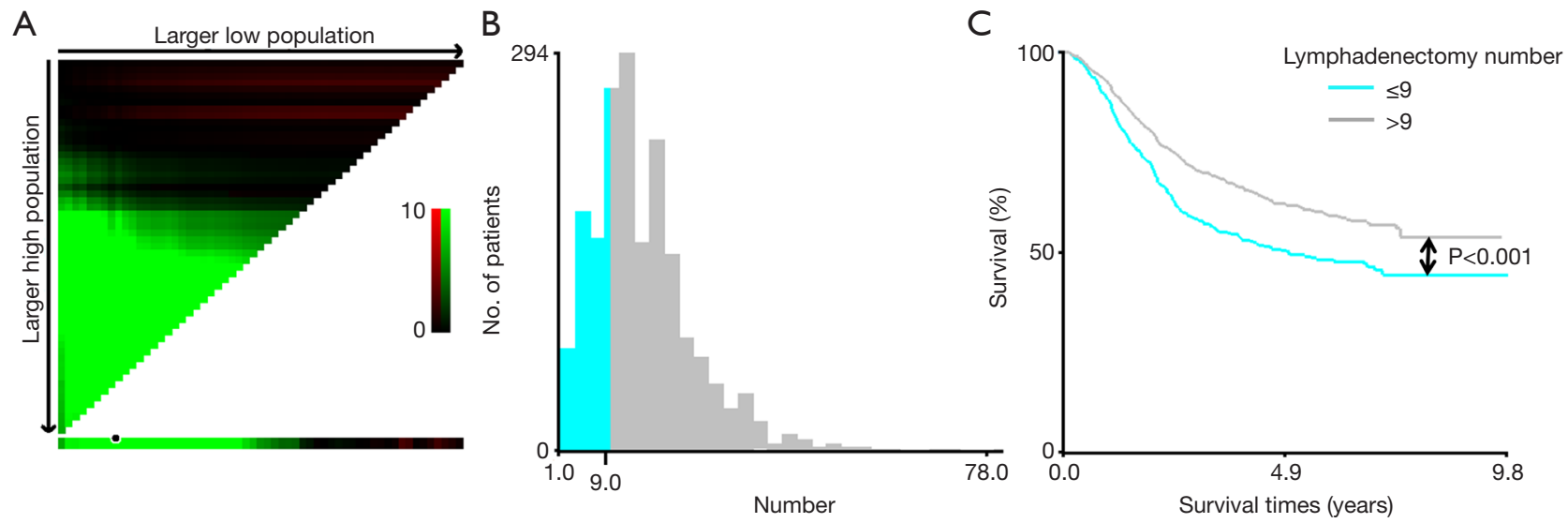

Figure $3 \mathrm{X}$-tile analysis of survival data from three tertiary esophagus centers (Training cohort). (A) The x-axis represents the cut-off value of all lymphadenectomy numbers from low to high (left to right) to define he high and low subsets. The stronger correlation between lymphadenectomy number at diagnosis and overall survival is, the brighter of pixels be presented. The plot shows the cut-off point of lymphadenectomy number is 9 through finding the brightest pixel (black circle marked), in which the training cohort is divided into high and low subsets. Green coloring means with the increase of lymphadenectomy number at diagnosis, the greater overall survival will be harvested directly. (B) The black circle in left panel highlighted the optimal cut-off point in histogram of the entire cohort. The blue and gray panel represent the patients with $\leq 9$ lymph nodes and $>9$ lymph nodes resected, respectively. (C) Among patients in training cohort, those with $>9$ lymph nodes resected had significantly better prognosis when compared with patients with $\leq 9$ lymph nodes resected $(\mathrm{P}<0.001)$.

16.37; SD 9.23) and 14 (range, 1-87; mean 15.24; SD 9.90), respectively $(\mathrm{P}=0.068)$. Among patients in training cohort, the 13,22, and 22 were considered as the lowest numbers of lymphadenectomy for stages T1, T2, and T3, respectively. Nevertheless, in validation cohort, the lowest number was 14, 7, and 10 for stages T1, T2, and T3, respectively.

\section{Nomogram for training cohort and for validation cobort}

In order to further test the feasibility of resecting at least 9 lymph nodes for N0 thoracic ESCC patients, nomogram for predicting 3-and 5-year survival in patients of the validation cohort was constructed based on the results of the Cox proportional hazard regression analysis. The nomogram showed that $\mathrm{T}$ stage made the largest contribution to prognosis, followed by tumor location, and the lymphadenectomy number group ranked the third. Differentiation showed the smallest effect on the survival rate (Figure 5). The coefficients are used to assign the score of these independent factors. Finally, the sum of these scores can be used to predict the probability of OS in 3 and 5 years. With regard to the discrimination of the nomogram, the C-index for training cohort and for validation cohort was 0.623 (95\% CI: 0.619-0.654) and 0.643 (95\% CI: $0.633-0.676$ ), respectively, and the sensitivity as well as 1 - specificity was $80.7 \%$ and $72.5 \%$ in training cohort and $78.2 \%$ and $63.0 \%$ in validation cohort. A calibration plot of the nomogram is presented in Figure 6, which shows that the predicted 3-and 5-year survival probabilities for both cohorts agreed well with the real-world observations.

\section{Discussion}

In this study, we evaluated the prognostic value of resected lymph nodes number in patients with thoracic ESCC patients in N0 stage. Lymph node metastasis has been one of the important prognostic factors in patients with EC. Surgeries are recommended for patients with positive lymph nodes, and neoadjuvant therapy combined with surgery are more popular treatment strategies nowadays. For node-negative patients and patients with earlystage EC, endoscopic interventional or limited surgical procedures are becoming a new trend. However, lymph node-negative status cannot be considered as the absence of lymph node metastasis. Indeed, previously published research provided evidence that lymph node metastasis was present in over $40 \%$ of submucosal carcinomas even though they were lymph node-negative histologically, and the malignant potential of nodal micrometastasis could be 
Table 3 Univariate and multivariate Cox regression analyses of thoracic ESCC patients without lymph node metastasis in two cohorts

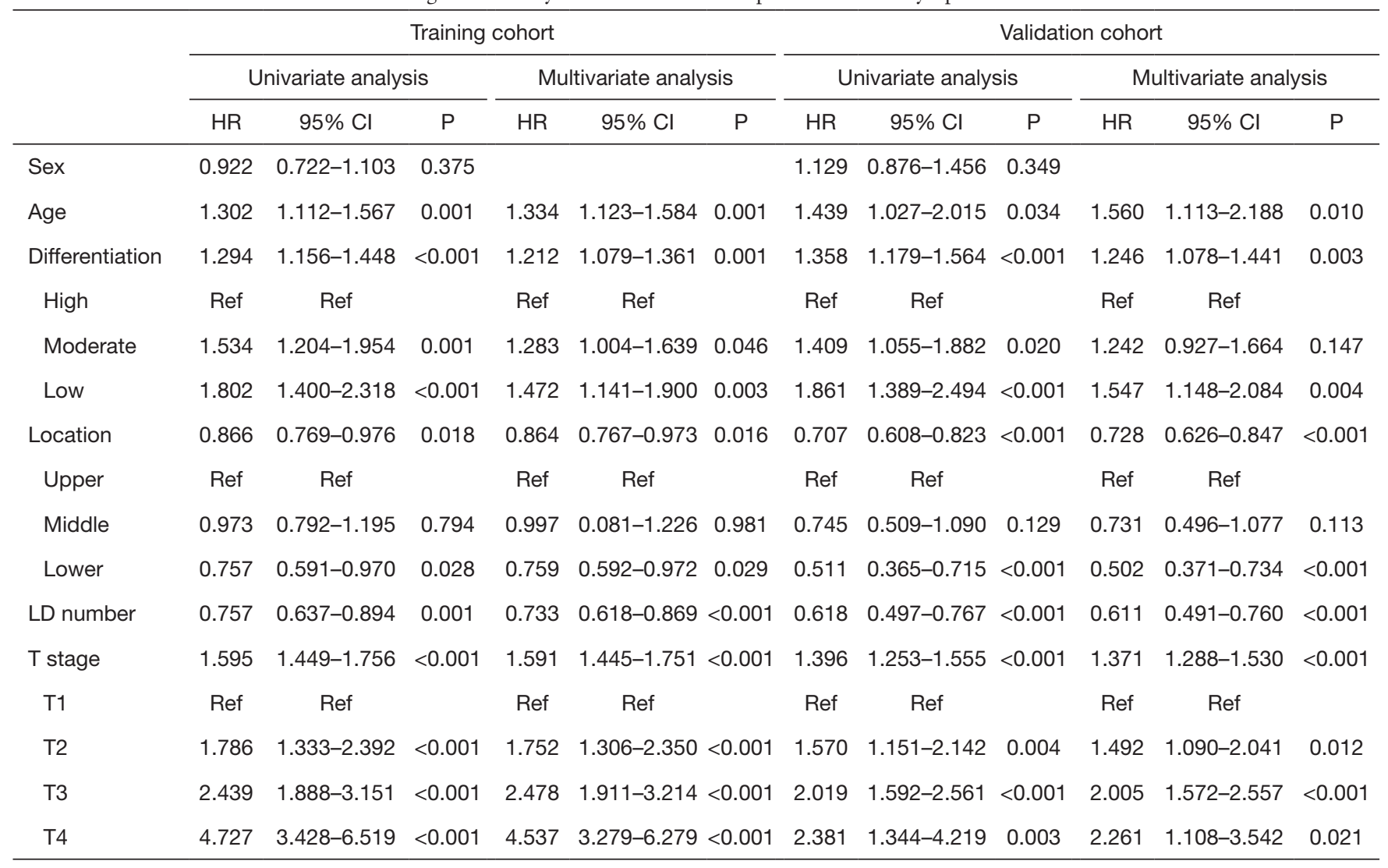

ESCC, esophageal squamous cell carcinoma; SEER, Surveillance, Epidemiology, and End Results; LD number, lymphadenectomy number; HR, hazard ratio; 95\% Cl, 95\% confidence interval; Ref, reference.

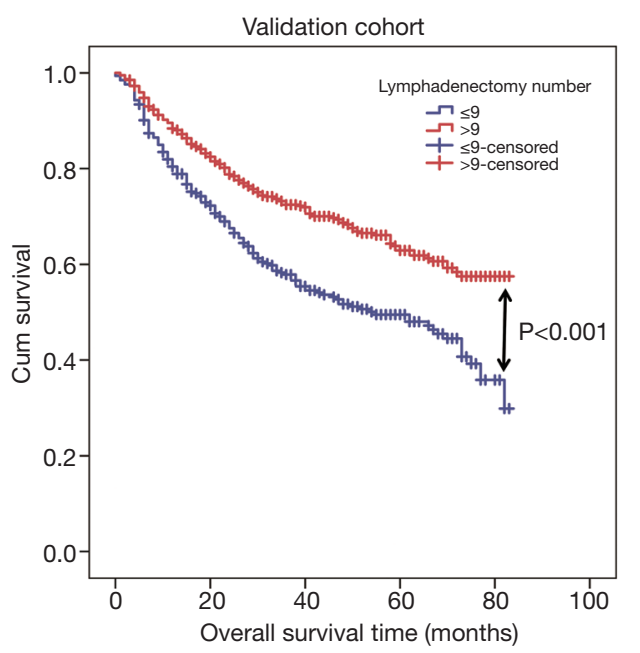

Figure 4 Prognostic impact of number 9 for resected lymph nodes in Surveillance, Epidemiology, and End Results (SEER) cohort. Overall survival in validation cohort with $>9$ lymph nodes resected was significantly better than that in patients with $\leq 9$ lymph nodes resected $(\mathrm{P}<0.001)$. detected immunohistochemically $(15,16,21,22)$. Prenzel et al. (23) reviewed 69 patients with pT1 stage cancer of esophagus and found that the prognosis in $\mathrm{pN} 0$ patients with micrometastasis was significantly worse than in those without micrometastasis (5-year survival: $57 \%$ vs. $82 \%$, $\mathrm{P}=0.002)$. Meanwhile, lamina muscularis mucosa was also confirmed as the barrier as lymphatic channels were only found in the submucosa like gastric cancer, which support for lymph node micrometastasis in patients of $\mathrm{pT} 1 \mathrm{~b}$ stage $(23,24)$. Therefore, the radical lymphadenectomy is necessary for node-negative patients, especially for those with cancers restricted to submucosa, in order to eliminate the lymph node micrometastasis. Nevertheless, the recommended number of lymph node harvest during esophagectomy for node-negative patients is unclear.

Regarding the extent of lymphadenectomy, many scholars have explored the role of the lowest number for lymphadenectomy and confirmed the effectiveness of more extensive lymphadenectomy to improve survival rates 


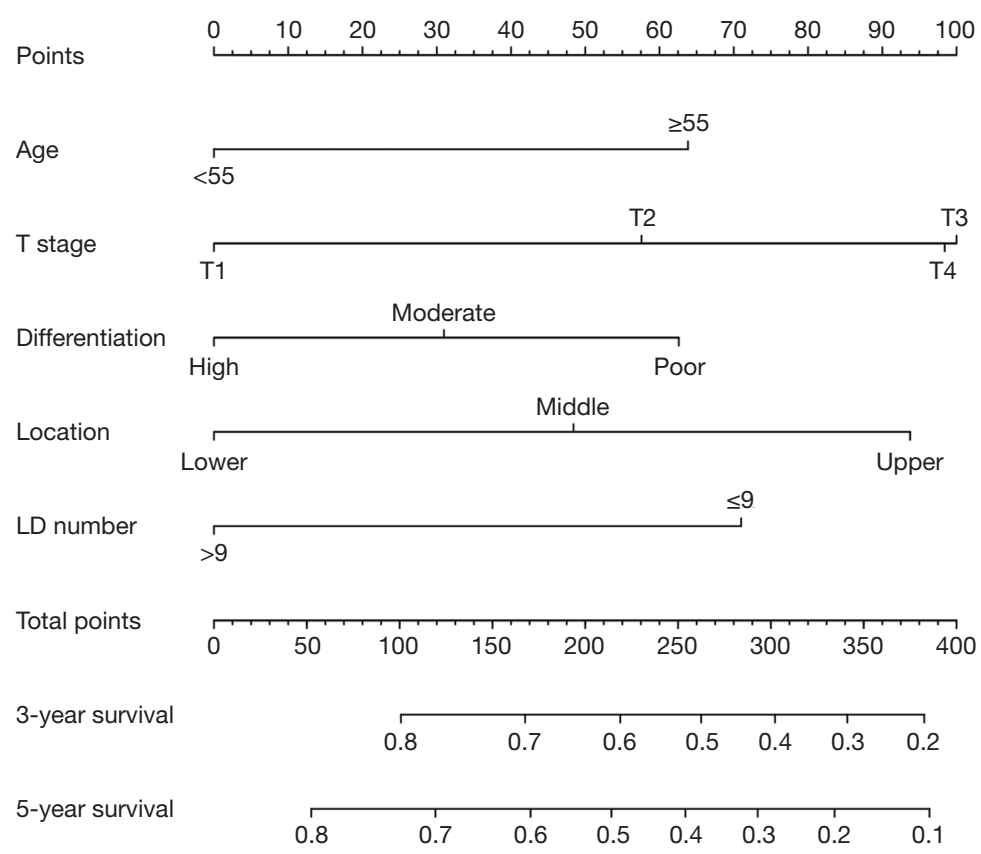

Figure 5 Prognostic nomogram for Surveillance, Epidemiology, and End Results (SEER) cohort. LD number, lymphadenectomy number.

A
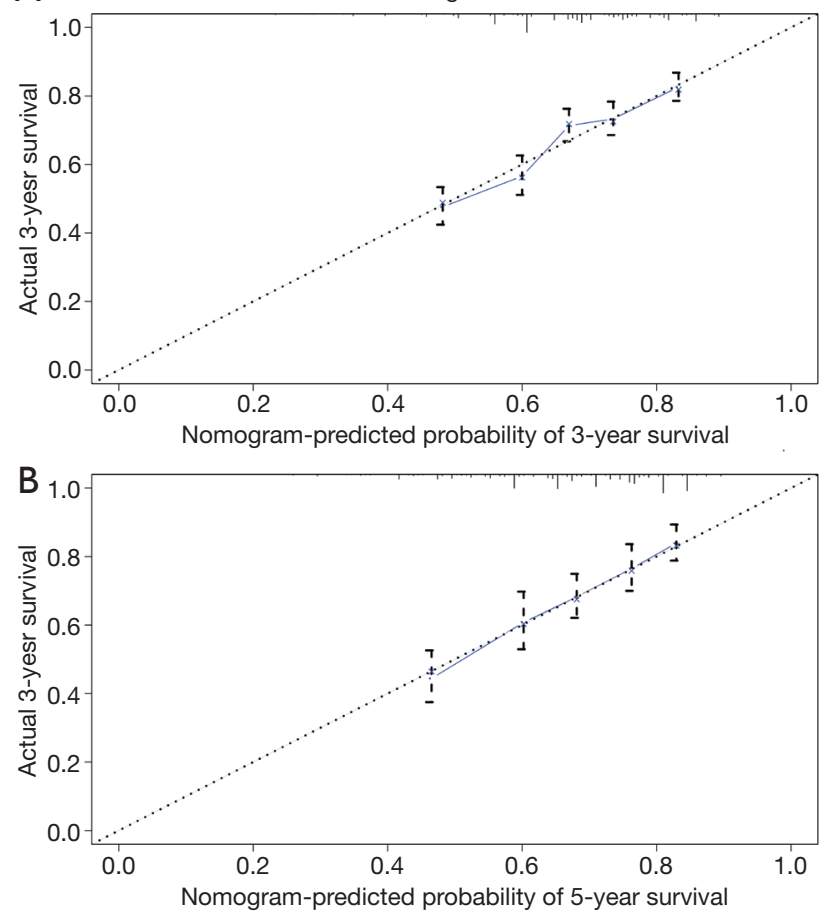

C
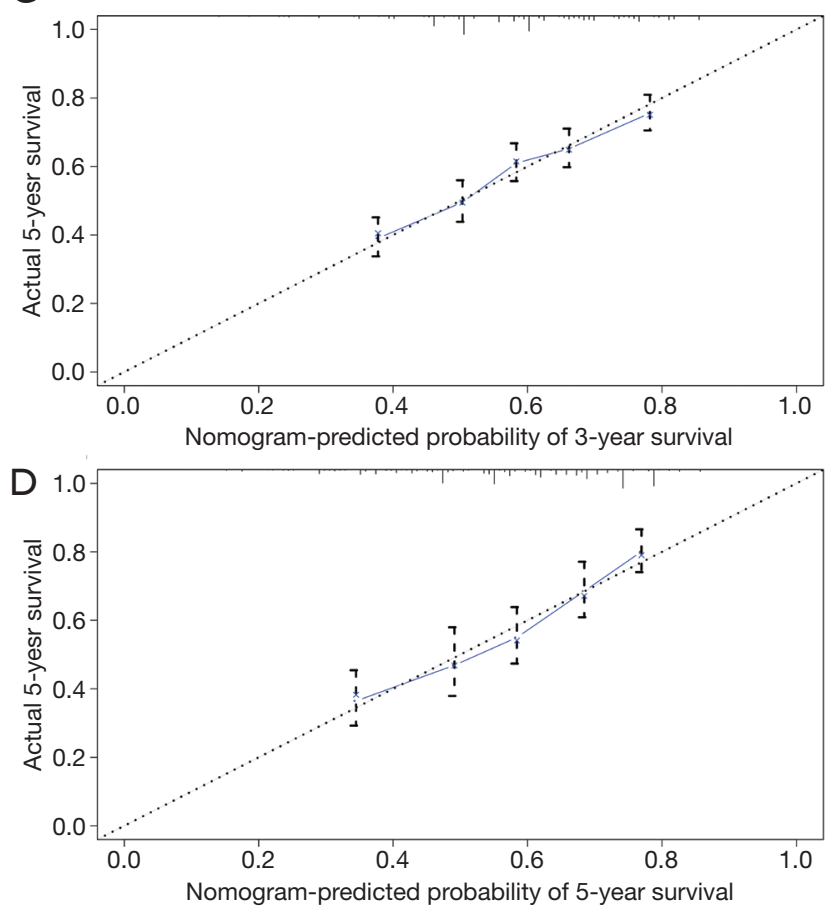

Figure 6 The calibration curves for predicting thoracic esophageal squamous cell carcinoma (ESCC) patient survival at (A) 3-year and (B) 5-year in Training cohort, and at (C) 3-year and (D) 5-year in Surveillance, Epidemiology, and End Results (SEER) cohort. Nomogrampredicted survival is plotted on the $\mathrm{x}$-axis; actual survival is plotted on the $\mathrm{y}$-axis. 
$(7,8,13)$. However, it is still controversial whether better survival was related to the higher number of harvested lymph nodes. On the basis of the worldwide data, the AJCC cancer staging manual 8th ed recommends the optimum lymphadenectomy for maximal survival following the simple rule of resecting 10 regional lymph nodes for pT1 cancers, 20 for $\mathrm{pT} 2$, and $\leq 30$ for $\mathrm{pT}$. Nevertheless, van der Schaaf et al. (11) reviewed 1,044 patients who had undergone the esophagectomy and found different results. Patients with 7-15 nodes removed and/or 16-114 nodes removed did not have a decrease in the 5 -year mortality rate when compared with those who had $<7$ nodes removed ( $\mathrm{HR}=1.13,95 \% \mathrm{CI}$ : $0.95-1.35$ and $\mathrm{HR}=1.17,95 \% \mathrm{CI}: 0.94-1.46$, respectively), especially in early $\mathrm{T}$ stages (Tis-T1) (HR $=1.53,95 \% \mathrm{CI}$ : 1.13-2.06). The same results were also shown in Lagergren et al. study (12). While many studies focused on the extent of lymphadenectomy, the exact number of lymph node dissection in the context of the presence or absence of lymph node metastasis was not addressed separately. Therefore, we specifically explored the number of lymph node harvest in $\mathrm{pN} 0$ patients in this study.

In our study, both training cohort and validation cohort have revealed an independent association between number of lymph node harvested and survival in node-negative thoracic ESCC patients. Obviously, more resected lymph nodes were correlated with the better prognosis, which was observed in subgroups stratified by age, sex, tumor location, differentiation and $\mathrm{T}$ stage in both cohorts. In terms of the relationship between the lowest number for lymphadenectomy and survival, 9 was identified as the minimum required for lymphadenectomy for nodenegative thoracic ESCC patients. The threshold number of 9 was examined and validated in the validation cohort and the prognostic differences remained significant. This result agreed with those shown in previous studies suggesting that high number of lymph nodes harvested was associated with better survival $(25,26)$. Furthermore, a recent metaanalysis that included 26 studies, also demonstrated that the OS was significantly improved in the high lymph node yield group ( $\mathrm{HR}=0.81 ; 95 \% \mathrm{CI}$ : $0.74-0.87 ; \mathrm{P}<0.01)(27)$. One possible reason for these results is that a higher number of resected lymph nodes guaranteed the radical clearance of metastasized lymph nodes, even for the lymph node micrometastasis (23-25). Meanwhile, the number for lymphadenectomy represents the adequacy of surgery, pathology and institutional care, affecting the treatment outcome eventually (26).

With regard to the validation cohort, we have found that 10 was the threshold number for lymphadenectomy. When stratified by $\mathrm{T}$ stage, firstly, no significant differences in lymphadenectomy number were found between two cohorts from T1 to T3 stages. Subsequently, we identified 13 in training cohort as the lowest number for T1 stage when compared with 14 in validation cohort. However, in training cohort and validation cohort, 22 and 7, respectively, were regarded as the lowest numbers for $\mathrm{T} 2$, and 22 was considered to be the lowest number for T3, compared with 10 in validation cohort. The lowest number for lymphadenectomy in training cohort for every $\mathrm{T}$ stage well corresponded with the guidance of the AJCC cancer staging manual, $8^{\text {th }}$ edition (6); moreover, the deeper tumor infiltration was, the highest was the incidence of lymph node micrometastasis even though the patient was node-negative, and a more radical lymphadenectomy was required in such circumstances $(7,10)$. Recently, Xia et al. (9) firstly examined the relationship between the number for lymphadenectomy and the accuracy of $\mathrm{N}$ staging and survival in EC patients, and reported the lowest number for lymphadenectomy in ESCC patients and N negative patients was 14 and 13, respectively, which is similar to our results; however, the ESCC patients in their study also included the patients with positive lymph nodes and $\mathrm{N}$ negative patients also included adenocarcinoma patients; therefore, the ESCC patients in N0 stage were not analyzed in their study.

The nomogram was applied in our study as it is an established model used to estimate the prognosis of cancer patients and it has been carried out in many malignant tumors $(25,28)$. The prognostic nomogram we constructed for both cohorts showed the acceptable agreement between the prediction probabilities and actual observations in terms of survival rate. Additionally, nomogram of validation cohorts seemed more agreeable than that in training cohort with regard to the least lymphadenectomy of 9 . Meanwhile, the specific survival rate for specific ESCC patient could also be estimated with the nomogram with regard to the included clinicopathological factors, which, to a degree, made it easier for surgeons to tailor personalized treatment scheme for patients (29).

There are also some limitations in our study. First, the low $\mathrm{C}$-index value suggested poor predictive value of the model, however, it can be explained as follows: on the one hand, this study is mainly focused on the optimal LD number for pN0 patients, therefore, patients with metastatic lymph nodes were ruled out initially, resulting in the included patients with single lymph node status. Besides, owing to the validation cohort was extracted from 
the SEER database, meanwhile, we had to exclude the patients with positive lymph nodes, distant metastasis and the patients received the neo- or adjuvant therapy followed by the exclusion criteria, therefore, the number of clinical variables we can get from SEER database were limited. In order to ensure the homogeneity and uniformity of comparison between two cohorts, the clinical variables we finally collected were based on the SEER database. Therefore, limited number of covariates included into the regression model may attribute the low predictive value, we do believe the 9 is an instructive number of $\mathrm{LD}$ in $\mathrm{pN} 0$ ESCC patients through our study. Of course, more studies for confirming our results are warranted. Second, in order to mainly focus on studying the impact of lymph node harvest for node-negative ESCC patients, we excluded the patients receive induction neoadjuvant and adjuvant therapy from this study, thus the findings cannot be generalized to those treated with pre-and postoperative therapy. Third, although this study was a multi-center retrospective study, selection bias existed in the choice of surgical approaches and follow-up could not be avoided.

In conclusion, based on three tertiary esophagus centers, our analyses showed that a large number for lymphadenectomy was significantly associated with better survival in node-negative thoracic ESCC patients. Therefore, 9 was we got as the lowest number for lymphadenectomy in pN0 ESCC patients as confirmed through the validation of data obtained from SEER database, and our result should be confirmed further.

\section{Acknowledgments}

Funding: This study was funded by Chengdu Science \& Technology Bureau, Grant 2019-GH02-00080-HZ.

\section{Footnote}

Reporting Checklist: The authors have completed the TRIPOD reporting checklist. Available at https://dx.doi. org/10.21037/jgo-20-371

Data Sharing Statement: Available at https://dx.doi. org/10.21037/jgo-20-371

Conflicts of Interest: All authors have completed the ICMJE uniform disclosure form (available at https://dx.doi. org/10.21037/jgo-20-371). All authors report that this study was funded by Chengdu Science \& Technology Bureau,
Grant 2019-GH02-00080-HZ. The authors have no other conflicts of interest to declare.

Ethical Statement: The authors are accountable for all aspects of the work in ensuring that questions related to the accuracy or integrity of any part of the work are appropriately investigated and resolved. The study was conducted in accordance with the Declaration of Helsinki (as revised in 2013), and was approved by the human participants' committee of West China Hospital of Sichuan University (No. 2021762A), Shantou University Medical College (No. 2020-094), and Sun Yat-sen University Cancer Centre (No. 2013B021800163, Science and Technology Plan Projects of Guangdong Province of China), and all patients were informed of the risk of the operation. The permission to use of resected specimens and the written consents were obtained preoperatively.

Open Access Statement: This is an Open Access article distributed in accordance with the Creative Commons Attribution-NonCommercial-NoDerivs 4.0 International License (CC BY-NC-ND 4.0), which permits the noncommercial replication and distribution of the article with the strict proviso that no changes or edits are made and the original work is properly cited (including links to both the formal publication through the relevant DOI and the license). See: https://creativecommons.org/licenses/by-nc-nd/4.0/.

\section{References}

1. Global Burden of Disease Cancer Collaboration; Fitzmaurice C, Dicker D, et al. The Global Burden of Cancer 2013. JAMA Oncol 2015;1:505-27.

2. Ferlay J, Soerjomataram I, Dikshit R, et al. Cancer incidence and mortality worldwide: sources, methods and major patterns in GLOBOCAN 2012. Int $J$ Cancer 2015;136:E359-86.

3. Ping YM, Zhang YD, Du QX, et al. Surgical treatment experiences of 20000 cases of esophageal and cardiac cancer. The First International Symposium on Esophageal Cancer and the Seventh National Symposium on Esophageal Cancer in China, 2005;167-171.

4. Rice TW, Ishwaran H, Hofstetter WL, et al. Esophageal Cancer: Associations With (pN+) Lymph Node Metastases. Ann Surg 2017;265:122-9.

5. Gertler R, Stein HJ, Schuster T, et al. Prevalence and topography of lymph node metastases in early esophageal and gastric cancer. Ann Surg 2014;259:96-101. 
6. Amin MB, Edge S, Greene FL, et al. AJCC Cancer Staging Manual (M). 8th ed. New York: Springer, 2017:185-202.

7. Darling G. The role of lymphadenectomy in esophageal cancer. J Surg Oncol 2009;99:189-93.

8. Bogoevski D, Onken F, Koenig A, et al. Is it time for a new TNM classification in esophageal carcinoma? Ann Surg 2008;247:633-41.

9. Xia W, Liu S, Mao Q, et al. Effect of lymph node examined count on accurate staging and survival of resected esophageal cancer. Thorac Cancer 2019;10:1149-57.

10. Jamieson GG, Lamb PJ, Thompson SK. The role of lymphadenectomy in esophageal cancer. Ann Surg 2009;250:206-9.

11. van der Schaaf M, Johar A, Wijnhoven B, et al. Extent of lymph node removal during esophageal cancer surgery and survival. J Natl Cancer Inst 2015;107:djv043.

12. Lagergren J, Mattsson F, Zylstra J, et al. Extent of Lymphadenectomy and Prognosis After Esophageal Cancer Surgery. JAMA Surg 2016;151:32-9.

13. Kelty CJ, Kennedy CW, Falk GL. Ratio of metastatic lymph nodes to total number of nodes resected is prognostic for survival in esophageal carcinoma. J Thorac Oncol 2010;5:1467-71.

14. Peyre CG, Hagen JA, DeMeester SR, et al. The number of lymph nodes removed predicts survival in esophageal cancer: an international study on the impact of extent of surgical resection. Ann Surg 2008;248:549-56.

15. Zingg U, Montani M, Busch M, et al. Prognostic influence of immunohistochemically detected lymph node micrometastasis and histological subtype in $\mathrm{pN} 0$ oesophageal cancer. Eur J Surg Oncol 2009;35:593-9.

16. Heeren PA, Kelder W, Blondeel I, et al. Prognostic value of nodal micrometastases in patients with cancer of the gastro-oesophageal junction. Eur J Surg Oncol 2005;31:270-6.

17. Thompson SK, Ruszkiewicz AR, Jamieson GG, et al. Isolated tumor cells in esophageal cancer: implications for the surgeon and the pathologist. Ann Surg 2010;252:299-306.

18. de Boer M, van Deurzen CH, van Dijck JA, et al. Micrometastases or isolated tumor cells and the outcome of breast cancer. N Engl J Med 2009;361:653-63.

19. Surveillance, Epidemiology, and End Results (SEER) Program (www.seer.cancer.gov) Research Data (19752016), National Cancer Institute, DCCPS, Surveillance Research Program, released April 2019, based on the November 2018 submission.
20. Camp RL, Dolled-Filhart M, Rimm DL. X-tile: a new bio-informatics tool for biomarker assessment and outcome-based cut-point optimization. Clin Cancer Res 2004;10:7252-9.

21. Bollschweiler E, Baldus SE, Schröder W, et al. High rate of lymph-node metastasis in submucosal esophageal squamous-cell carcinomas and adenocarcinomas. Endoscopy 2006;38:149-56.

22. Izbicki JR, Hosch SB, Pichlmeier U, et al. Prognostic value of immunohistochemically identifiable tumor cells in lymph nodes of patients with completely resected esophageal cancer. N Engl J Med 1997;337:1188-94.

23. Prenzel KL, Hölscher AH, Drebber U, et al. Prognostic impact of nodal micrometastasis in early esophageal cancer. Eur J Surg Oncol 2012;38:314-8.

24. Hölscher AH, Drebber U, Mönig SP, et al. Early gastric cancer: lymph node metastasis starts with deep mucosal infiltration. Ann Surg 2009;250:791-7.

25. Cao J, Yuan P, Wang L, et al. Clinical Nomogram for Predicting Survival of Esophageal Cancer Patients after Esophagectomy. Sci Rep 2016;6:26684.

26. Fujita H, Kakegawa T, Yamana H, et al. Lymph node metastasis and recurrence in patients with a carcinoma of the thoracic esophagus who underwent three-field dissection. World J Surg 1994;18:266-72.

27. Visser E, Markar SR, Ruurda JP, et al. Prognostic Value of Lymph Node Yield on Overall Survival in Esophageal Cancer Patients: A Systematic Review and Meta-analysis. Ann Surg 2019;269:261-8.

28. Deng W, Wang Q, Xiao Z, et al. A prognostic nomogram for overall survival after neoadjuvant radiotherapy or chemoradiotherapy in thoracic esophageal squamous cell carcinoma: a retrospective analysis. Oncotarget 2017;8:41102-12.

29. Liang W, Zhang L, Jiang G, et al. Development and validation of a nomogram for predicting survival in patients with resected non-small-cell lung cancer. J Clin Oncol 2015;33:861-9.

Cite this article as: Shang QX, Yang YS, Xu LY, Yang H, Li Y, Li Y, Wu ZY, Fu JH, Yao XD, Xu XE, Wu JY, Chen LQ. Prognostic impact of lymph node harvest for patients with node-negative esophageal squamous cell carcinoma: a largescale multicenter study. J Gastrointest Oncol 2021;12(5):19511962. doi: 10.21037/jgo-20-371 